\title{
Pulmonary fibrosis in an individual occupationally exposed to inhaled indium-tin oxide
}

\author{
S. Homma*, A. Miyamoto*, S. Sakamoto*, K. Kishi*, N. Motoi ${ }^{*}$ and K. Yoshimura*
}

ABSTRACT: Despite the increasing industrial use of indium-tin oxide (ITO) to manufacture flatpanel displays, such as liquid-crystal displays or plasma display panels for televisions, little is known about the potential health hazard induced by occupational exposure to indium compounds.

The current study describes a case of fibrotic lung disease that developed after a 4-yr exposure to ITO. The pathology of the lung demonstrated pulmonary fibrosis with the presence of cholesterol granulomas.

In conclusion, more attention needs to be paid to the possible toxic effects of indium compounds, and maximum healthcare measures should be taken to protect industry workers from these toxicities.

KEYWORDS: Cholesterol granuloma, human, indium-tin oxide, lung injury, pulmonary fibrosis

I ndium belongs to Group III in the periodic table and is mainly used in the manufacture of flat-panel displays, such as liquid-crystal displays or plasma display panels for television screens, computer screens and video monitors $[1,2]$. In these displays, indium-tin oxide (ITO) is utilised for transparent conductive films. In light of the recent growth of the market due to the increasing use of liquid-crystal displays in personal computer screens and plasma display panels in wall-mounted televisions, the demand for ITO has risen sharply. As industrial consumption of ITO rises, the potential health hazard caused by occupational exposure to indium compounds has been attracting much more attention than before. However, in the literature, there have been a limited number of reports of pulmonary toxicity by ITO, including only one autopsied human case with occupational exposure to indium compounds [3], and a few preliminary animal studies on the lung toxicity of indium [4-9]. In the present study, a previously healthy young male with lung injury, probably caused by inhalation of ITO, is described.

\section{CASE PRESENTATION}

In January 2002, a 30-yr-old male engineer visited the current authors' hospital (Dept of Respiratory
Medicine, Respiratory Centre, Toranomon Hospital, Tokyo, Japan) complaining of dry cough and exertional dyspnoea, which he had been experiencing since 1997 . He had a minimum history of cigarette smoking (3 cigarettes $\cdot$ day $^{-1}$ for 3 yrs). From 1994 to 1998, he had been exposed to indium compounds (i.e. ITO) as an aerosol while making transparent conductive films. Physical examination of the chest on admission revealed normal vesicular breath sounds and the absence of finger clubbing. Pulmonary function tests and arterial blood gas analysis in room air yielded normal values: a vital capacity of $4.18 \mathrm{~L}$ (93\% predicted), forced expiratory volume in one second of $3.07 \mathrm{~L}$ (73\% pred), residual volume (RV) of $2.20 \mathrm{~L}$ (measured by the gas dilution method; $158 \%$ pred), total lung capacity (TLC) of 6.38 L (109\% pred), RV/TLC ratio of $34 \%$, carbon dioxide diffusing capacity of the lung of $3.21 \mathrm{~mL} \cdot \mathrm{min}^{-1} \cdot \mathrm{kPa}(89 \%$ pred $), \mathrm{pH} 7.40$, arterial oxygen tension of $11.8 \mathrm{kPa}$, and arterial carbon dioxide tension of $5.3 \mathrm{kPa}$ [10]. Peripheral blood examination showed that the counts of leukocytes and red blood cells, as well as the level of C-reactive protein, were normal, while KL-6, as an indicator of active pneumonitis, rose slightly to $799 \mathrm{U} \cdot \mathrm{mL}^{-1}$ (normal values $<500 \mathrm{U} \cdot \mathrm{mL}^{-1}$ )

\section{AFFILIATIONS}

Depts of *Respiratory Medicine, Respiratory Center, and \#Pathology, Toranomon Hospital, Tokyo, Japan.

CORRESPONDENCE

S. Homma

Dept of Respiratory Medicine

Toranomon Hospital

Toranomon 2-2-2

Minato-ku

Tokyo

105-8470

Japan

Fax: 81335827068

E-mail: sa-homma@toranomon.gr.jp

Received:

February 022004

Accepted after revision:

June 172004 
[11]. The serum indium level measured by inductively coupled plasma mass spectrometry (Agilent Technologies, Palo Alto CA, USA) was high at $51 \mu \mathrm{g} \cdot \mathrm{L}^{-1}$ (normal values $<0.1 \mu \mathrm{g} \cdot \mathrm{l}^{-1}$ ) [12]. Chest roentgenogram on admission showed reticulonodular shadows in the right upper lung field. On chest computed tomography (CT), diffuse ground-glass opacities were present adjacent to the peripheral air space consolidation in the right upper lobe. Diffusely scattered centrilobular fine nodular densities in both lung fields associated with emphysematous low attenuation areas at both apexes were also seen (fig. 1). An oedematous, reddish mucosa in the entire bronchial tree was observed by means of flexible bronchoscopy. Bronchoalveolar lavage performed through the right $\mathrm{B} 5$ bronchus revealed a minor increase in lymphocytes: 300,000 cells $\cdot \mathrm{mL}^{-1}$ (macrophages $77.8 \%$, lymphocytes $18.8 \%$ (CD4+/CD8+ ratio of 1.14$)$, neutrophils $2.4 \%$, eosinophils $0.6 \%$, basophils $0.4 \%$ ).

The patient underwent video-assisted thoracoscopic surgery for the diagnosis of the lung lesion. The macroscopic appearance of the cut surface of both the right posterior segment of the upper lobe (S2) and the anterior segment of the lower lobe (S8) included diffusely small, solid yellowish lesions in the air space associated with subpleural
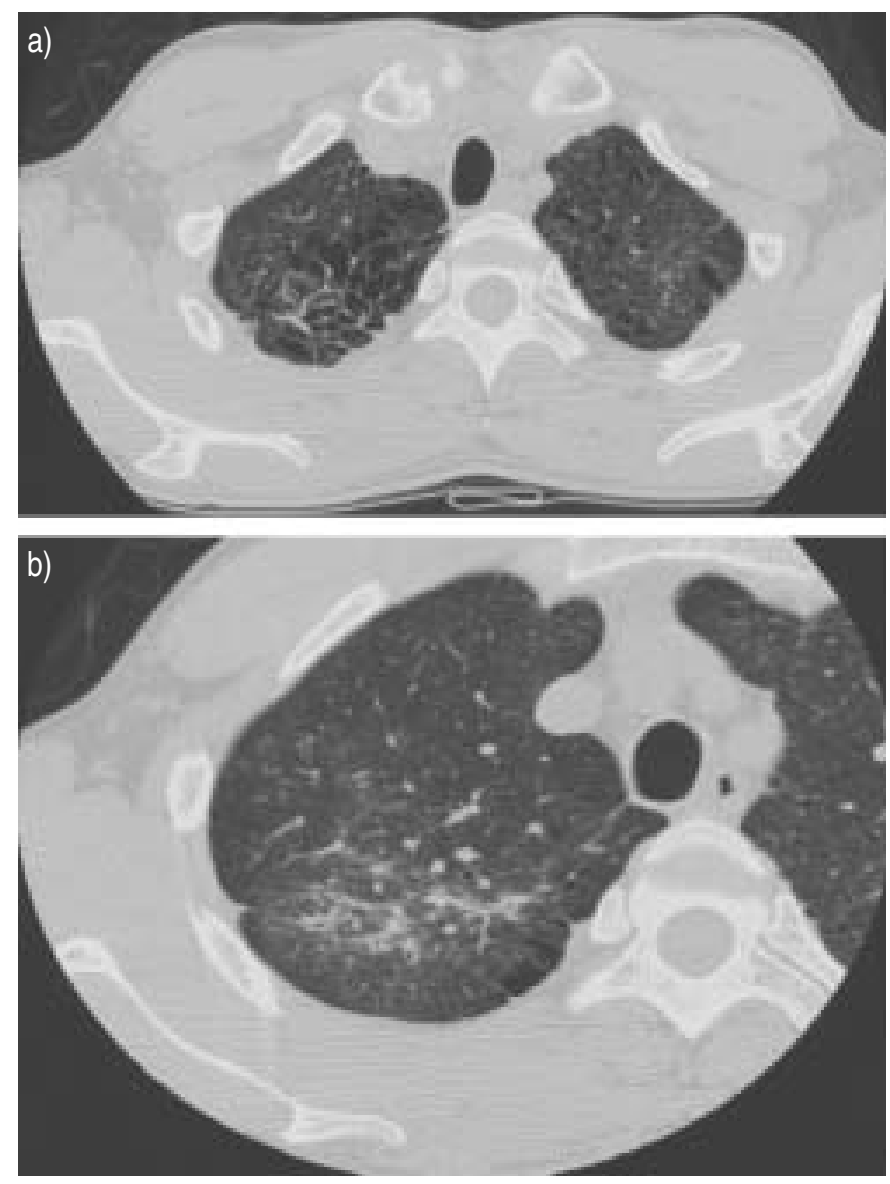

FIGURE 1. Chest computed tomography scan on admission. a) Diffusely scattered centrilobular fine nodular densities associated with emphysematous low attenuation areas in both apexes. b) Increased diffuse ground-glass opacities adjacent to peripheral air space consolidation in the right upper lung field. emphysematous changes. Upon microscopic examination at high magnification, these solid lesions appeared to correspond to proliferating peribronchial fibrous tissues in the centrilobular regions with numerous cholesterol clefts and foreign body-type giant cells containing brown particles (fig. 2). There were no lobuli without injury in either specimens from the right S2 and S8 segments. In addition to these findings, diffuse alveolitis with intra-alveolar accumulation of alveolar macrophages containing brown particles was observed. Electron probe X-ray microanalysis [13], field emission-scanning electron microscopy (Hitachi Co. Ltd., Tokyo, Japan), and energy dispersive X-ray analytical spectrometer (Kevex Sigma, Trento, Italy) studies on these lung tissues confirmed that the particles contained a large amount of indium $(61 \%)$ and a small amount of tin (4\%; fig. 3$)$.

Based on these pathological findings, the patient was diagnosed as having pulmonary fibrosis (with presence of cholesterol granulomas) and emphysema, very probably caused by inhalation of ITO. Since the patient's transfer to another section within the company in 1998, he has recovered from his subjective symptoms, and has been well without undergoing any treatment. The follow-up chest CT and blood gas analysis showed no progression of the pulmonary disease (not shown).

\section{DISCUSSION}

There have been a relatively limited number of reports in the literature describing pulmonary toxicities of inhaled indiumcontaining materials, such as indium arsenide or indium phosphide, in animals as well as humans [3-9]. However, only one report regarding experimental pulmonary toxicity of ITO has been published to date [4]. In the study by TANAKA et al. [4], the pulmonary toxic effect of ITO and indium phosphide was evaluated by repeated instillation into the trachea of hamsters. In the ITO group, the inflammatory response was milder than that observed in the indium phosphide group; for example, inflammatory foci seen in the lung were less prominent. However, in the same ITO group, numerous alveolar macrophages with expanded cytoplasm containing various amounts of brown ITO particles, necrotic cell debris or a small number of neutrophils were present within the alveolar septa, alveolar spaces or bronchiolar lumens. The formation of cholesterol granulomas was not observed, although cholesterol clefts were slightly present in that animal model. Since ITO contains a high fraction of indium (indium $74 \%$; tin $8 \%$ ), the main cause of the observed lung toxicity was probably indium.

Prior to the present report, only one human case of interstitial pneumonia caused by inhaled ITO particles has been documented by Homma et al. [3]. The patient described came from the same factory as in the current study and died of bilateral pneumothorax after a 4-yr illness. He had been engaged in production of transparent conductive films made of ITO for flat-panel displays. The autopsy demonstrated interstitial pneumonia with numerous and defined fine particles scattered throughout both lungs. In addition, his alveolar spaces were filled with red blood cells, fibrin, cholesterol clefts and alveolar macrophages with expanded cytoplasm containing fine particles. Intrapulmonary deposition of indium and tin was shown by $\mathrm{X}$-ray energy spectrometry in the fine particles, as it was in the present case. The level of serum indium was extremely 

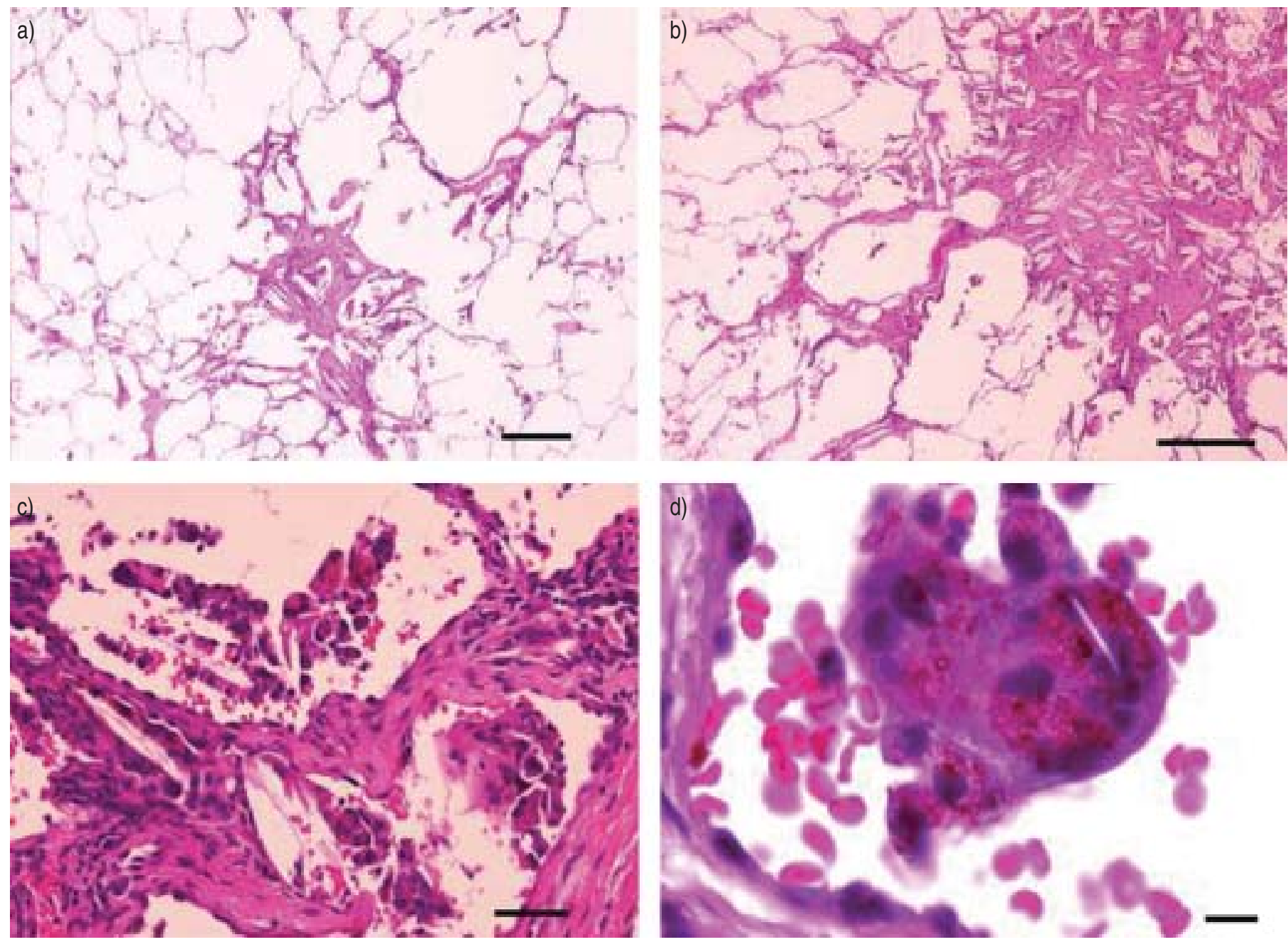

FIGURE 2. Microscopic evaluation of the video-assisted thoracic surgery biopsied lung specimen from the posterior segment of the right upper lobe. a) A proliferative peribronchial fibrosis with cholesterol clefts in the centrilobular region. b) Numerous cholesterol clefts in the interstitium and intra-alveolar spaces. c) Alveolitis associated with intra-alveolar accumulation of alveolar macrophages containing brown particles and cholesterol clefts. d) Magnified view of the intra-alveolar macrophages. Haematoxylin and eosin stain was used. Scale bars: a) $0.5 \mathrm{~mm}$, b) $0.25 \mathrm{~mm}, \mathrm{c}) 25 \mu \mathrm{m}$, d) $10 \mu \mathrm{m}$

high $\left(290 \mu \mathrm{g} \cdot \mathrm{L}^{-1}\right)$. Thus, the reported case was diagnosed as having interstitial pneumonia caused by the inhalation of ITO particles.

Likewise, the patient in the present report had been engaged in the same metal plant as the first case for $4 \mathrm{yrs}$, had a high serum indium level $\left(51 \mu \mathrm{g} \cdot \mathrm{L}^{-1}\right)$, and the pathological findings observed in the biopsied lung specimens, such as subpleural emphysema and the presence of numerous cholesterol granulomas, were reminiscent of those in the previously reported case. The patient in the current study has not yet developed pneumothorax, suggesting that emphysematous changes may be the late phenomenon of the progression of peribronchial fibrosis in the centrilobular regions.

ITO is a sintered alloy containing a large portion of indium oxide and a small portion of tin oxide. The mean count diameter of the ITO particles, as sampled in the workplace, was $1.0 \mu \mathrm{m}$. Both of these two cases were engaged in wet surface grinding of ITO target under the protection of wearing a dust mask. According to CHONAN and TAGUCHI [14], among
115 workers who were involved in this job in the same metal plant, 14 individuals revealed interstitial fibrosis on chest CT. Although the solubility of ITO particles both in vitro and in vivo has not been well documented, the high concentration of indium in the patients' sera indicated indium dissolution from ITO particles. Indium in the blood seems to be distributed to multiple organs and may cause chronic adverse health effects. For example, the first human case reported by HoMmA et al. [3] showed liver damage and splenomegaly. Testicular toxicity caused by intratracheal instillation of ITO was also demonstrated recently in hamsters [15]. However, the hazardous effects of the ITO particles appeared to be milder than those of indium phosphide in hamsters [4].

In these two cases, the common characteristic pathological feature is the presence of numerous cholesterol granulomas in both the alveolar spaces and the interstitium. In general, the formation of pulmonary interstitial and intra-alveolar cholesterol granulomas is the consequence of fibrous organisation of cholesterol crystals originating from lipid-laden macrophages 

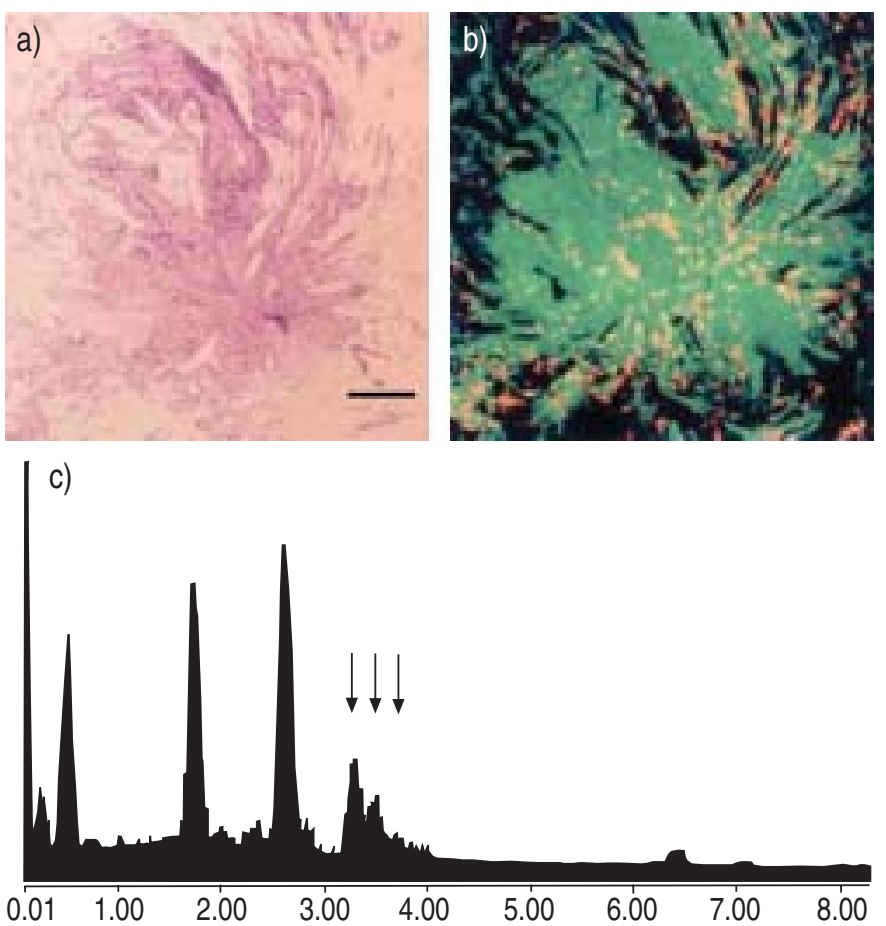

FIGURE 3. Microanalysis of the biopsied lung specimen. a) Alveolitis with intra-alveolar accumulation of alveolar macrophages and numerous cholesterol clefts (haematoxylin and eosin stain). Scale bar $=0.5 \mathrm{~mm}$. b) Electron probe X-ray microanalysis of a). The scattered small, white particles represent indium. c) Field emission-scanning electron microscopy and energy dispersive $\mathrm{X}$-ray analytical spectrometer studies on the lung tissues. Three peaks of indium are indicated by arrows.

or endogenous lipids in lipoid pneumonia [16]. Lipoid pneumonia itself can be divided into endogenous and exogenous diseases [17]. KAY et al. [18] also proposed that pulmonary cholesterol-ester granulomas in man might be the result of hyperplasia and desquamation of granular pneumocytes. However, in the present case, no apparent cause of either the exogenous or endogenous disease could be detected. In addition, histopathological examinations also showed no evidence of hyperplasia or desquamation of granular

\begin{tabular}{ll} 
TABLE 1 & $\begin{array}{l}\text { Results of semiquantitative analysis by electron } \\
\text { probe X-ray }\end{array}$ \\
In & 61.24 \\
$\mathbf{S i}$ & 12.12 \\
$\mathbf{O}$ & 8.68 \\
$\mathbf{A l}$ & 6.53 \\
$\mathbf{S n}$ & 4.32 \\
$\mathbf{F e}$ & 1.52 \\
$\mathbf{P}$ & 0.84 \\
$\mathbf{S}$ & 0.66 \\
$\mathbf{N a}$ & 0.45 \\
$\mathbf{C l}$ & 0.23 \\
$\mathbf{M g}$ & 0.20 \\
\hline
\end{tabular}

Results shown as \% component of particles (corresponds to fig. 3b). pneumocytes. Based on the above discussion, the current authors propose that the characteristic primary lesion caused by inhalation of ITO comprises the formation of interstitial and intra-alveolar cholesterol granulomas in the fibrotic lung, although the precise mechanism of the pathological processes responsible for the disease remains to be elucidated.

Finally, more comprehensive assessment of a population of indium-tin oxide workers is needed, and maximum healthcare measures should be taken to protect them from the potential toxicities of occupational exposure to indium compounds.

\section{ACKNOWLEDGEMENTS}

The authors would like to thank E. Suzuki of Niigata University, T. Gotoh and K. Matsumoto of Waseda University and $\mathrm{H}$. Taniguchi of the University of Tokyo for technical support in electron probe $\mathrm{X}$-ray microanalysis, field emission-scanning electron microscopy and energy dispersive X-ray analytical spectrometer studies. The authors also thank the Japan Industrial Safety and Health Association for measuring the serum indium level.

\section{REFERENCES}

1 Dupuis RD. Metalorganic chemical vapor depositin of IIIV semiconductors. Science 1984; 226: 623-629.

2 Fowler BA. Indium. In: Friberg L, Nordberg GF, Vouk VB, eds. Handbook on the toxicology of metals. 2nd Edn. Amsterdam, Elsevier, 1986; pp. 267-275.

3 Homma T, Ueno T, Sekizawa K, Tanaka A, Hirata M. Interstitial pneumonia developed in a worker dealing with particles containing indium-tin oxide. J Occup Health 2003; 45: 137-139.

4 Tanaka A, Hirata M, Omura M, et al. Pulmonary toxicity of indium-tin oxide and indium phosphide after intratracheal instillations into the lung of hamsters. J Occup Health 2002; 44: 99-102.

5 Blazka ME, Dixon D, Haskins E, Rosenthal G. Pulmonary toxicity to intratracheally administered indium trichloride in Fischer 344 rats. Fundam Appl Toxicol 1994; 22: 231239.

6 Blazka ME, Tepper JS, Dixon D, Winsett DW, O'Conner RW, Luster MI. Pulmonary response of Fischer 344 rats to acute nose-only inhalation of indium trichloride. Environ Res 1994; 67: 68-83.

7 Tanaka A, Hisanaga A, Hirata M, et al. Chronic toxicity of indium arsenide and indium phosphide to the lungs of hamsters. Fukuoka Acta Med 1996; 87: 108-115.

8 Yamazaki K, Tanaka A, Hirata M, et al. Long term pulmonary toxicity of indium arsenide and indium phosphide instilled intratracheally in hamsters. J Occup Health 2000; 42: 169-178.

9 Gottschling BC, Maronpot RR, Hailey JR, et al. The role of oxidative stress in indium phosphide-induced lung carcinogenesis in rats. Toxicol Sci 2001; 64: 28-40.

10 Ebina T, Kanagami H, Katsura T, Aonuma K, Shiroishi K. On the predicted value of pulmonary function tests in normal Japanese. Kokyu To Junkan 1958; 6: 999-1013.

11 Kohno N, Kyoizumi S, Awaya Y, Fukuhara H, Yamakido M, Akiyama M. New serum indicator of interstitial pneumonitis activity. Chest 1989; 96: 68-73. 
12 Chiba M. Concentrations of essential trace elements in blood and introduction of analytical techniques. Nippon Rinsho 1996; 54: 179-185.

13 Mangabeira P, Mushrifah I, Escaig F, Laffray D, Franca MGC, Galle P. Use of SIMS microscopy and electron probe X-ray microanalysis to study the subcellular localization of aluminium in Vicia faba roots cells. Cell $\mathrm{Mol}$ Biol 1999; 45: 413-422.

14 Chonan $\mathrm{T}$, Taguchi $\mathrm{O}$. The incidence of interstitial pneumonia patients in an ITO sputtering targets producing factory. Nihon Kokyuki Gakkai Zasshi 2004; 42: 185.
15 Omura M, Tanaka A, Hirata M, et al. Testicular toxicity evaluation of indium-tin oxide. J Occup Health 2002; 44: 105-107.

16 Fisher M, Roggli V, Merten D, Mulvihill D, Spock A. Coexisting endogenous lipoid pneumonia, cholesterol granulomas, and pulmonary alveolar proteinosis in a pediatric population. Pediatr Pathol 1992; 12: 365-383.

17 Wright BA, Jeffrey PH. Lipoid pneumonia. Semin Respir Infect 1990; 5: 314-321.

18 Kay JM, Heath D, Hasleton PS, Littler WA. Aetiology of pulmonary cholesterol-ester granulomas. $\mathrm{Br} J$ Dis Chest 1970; 64: 55-57. 